\title{
EDITORIAL
}

\section{Assessing life expectancy: our continuing}

Armen Aprikian, MD, FRCSC

Interim Editor-in-Chief CUAJ $\mathrm{n}$ counselling men with localized prostate cancer, we often attempt to estimate the probability of a 10-year life expectancy to decide whether definitive local therapy, with its associated morbidity, is warranted. In this issue of CUAJ, Jeldres and colleagues $^{1}$ ask the important question - do men treated for prostate cancer actually survive past 10 years? The authors present their study on the overall mortality of a large provincial cohort of men with prostate cancer who had undergone either radical prostatectomy or radiotherapy. This study is the first of its kind in Canada and the results are quite interesting and provocative. The results show that a significant proportion of men actually do not live beyond 10 years after therapy. Of note, only an estimated $35 \%$ of men treated with radiotherapy were alive 10 years after treatment. Not surprisingly, the study shows that men having undergone radiotherapy were older and sicker than those having had surgery. This finding suggests that many men treated with radiotherapy for prostate cancer suffer from comorbid conditions and die of other causes, indicating perhaps that radiotherapy (or any therapy for that matter) was not necessary. On the other hand, it is possible that for some men the treatment delayed the onset of symptoms or avoided the toxicity of androgen ablation later, even if they died from other causes before the 10-year mark.

As urologists, we are usually the ones to discuss the various treatment modalities for localized prostate cancer. Accordingly, virtually all of the men in this study having had radiotherapy had an initial discussion with a urologist. Urologists recommend radiotherapy over surgery because of patient age and comorbid conditions. Therefore, it is conceivable that urologists recommend radiotherapy to patients even though they may believe that life expectancy may be limited. Consequently, radiation oncologists find themselves facing patients with significant comorbid conditions who have been told that radiotherapy is better for them.

It is true that our ability to assess life expectancy is not great. Jeldres and colleagues' study clearly shows that we must significantly improve in this domain. We have justifiably been focused for some time on not overtreating men with localized prostate cancer for fear of morbidity in the face of insignificant cancer. This study, however, clearly illustrates the point that we are probably overtreating men who also have poor life expectancy.

\section{Reference}

1. Jeldres C, Suardi N, Perrotte P, et al. Survival after radical prostatectomy and radiotherapy for prostate cancer: a population-based study. Can Urol Assoc J 2009;3:13-21. 